\title{
Roles of SEA-expressing Staphylococcus aureus, isolated from an atopic dermatitis patient, on expressions of human B-defensin-2 and inflammatory cytokines in $\mathrm{HaCaT}$ cells
}

\author{
JAE-WE CHO, SUN-YOUNG CHO and KYU-SUK LEE
}

\author{
Department of Dermatology, School of Medicine, Keimyung University, \\ 194 DongSan-Dong Jung-Gu, Daegu 700-712, South Korea
}

Received November 14, 2008; Accepted December 29, 2008

DOI: 10.3892/ijmm_00000135

\begin{abstract}
Atopic dermatitis (AD) shows an increased susceptibility to Staphylococcus aureus infection partly due to decreased expression of human B-defensin-2 (HBD-2). Interestingly, it was reported that the nasal carrier $S$. aureus down-regulates the expression of HBD-2 and -3, thereby the carrier strains of $S$. aureus retain an advantage to epithelial colonization and infection. In this study, we tried to isolate and characterize $S$. aureus from an AD patient, with recurrent oozing on his face. We studied the increased expression of inflammatory cytokines, such as IL-1ß, $-6,-8$, and TNF- $\alpha$ in $S$. aureus treated-HaCaT cells, which are mediated by secreting superantigens (SAgs), structural component, or both. In addition, we investigated whether the SAgs from $S$. aureus can downregulate the expression of HBD-2 in HaCaT cells making favorable conditions for colonization on skin. Our data showed that the isolated $S$. aureus has the exotoxin gene, sea exotoxin. The SEA producing-S. aureus induced the expression of IL-1ß, $-6,-8$ cytokines, and TNF- $\alpha$ in HaCaT cells. The expression of HBD-2 was increased in $S$. aureus-treated HaCaT cells. Furthermore IL-8 was also induced by the structure component of $S$. aureus. Taken together, the SEA producing S. aureus induced the up-regulation of pro-inflammatory cytokines as well as HBD-2, thereby resulting in induction of the persistent eczematous skin lesions in AD. Thus, our data may give insight into understanding the pathogenesis by which $S$. aureus induces and aggravates eczematous skin lesions in $\mathrm{AD}$.
\end{abstract}

\section{Introduction}

Atopic dermatitis (AD) is an inflammatory skin disease with a course of remission and exacerbation (1). When AD is exacer-

Correspondence to: Dr Kyu-Suk Lee, Department of Dermatology, Keimyung University School of Medicine, 194 DongSan-Dong Jung-Gu, Daegu 700-712, South Korea

E-mail: janylove99@hanmail.net

Key words: atopic dermatitis, Staphylococcal aureus, superantigen, cytokines, human $\beta$-defensin-2 bated by several factors including infection and dryness, acute erythematous eczema lesions are sometimes observed in the skin, characterized by erythema, oozing and crusting $(2,3)$.

Staphylococcus aureus expressing superantigens (SAgs) induce eczematoid skin reactions in AD patients $(4,5)$. Over half of the S. aureus strains isolated from AD skin exhibited the secretion of superantigen toxins, including staphylococcal enterotoxins A and B (SEA, SEB), and toxic shock syndrome toxin-1 (TSST-1), which induces the pro-inflammatory effects through secretion of inflammatory cytokines such as IL-1ß, -6, -8 , and TNF- $\alpha$. These cytokines induce persistent eczema in AD (6-8). However, it is still unknown if the increased expression of pro-inflammatory cytokines are mediated by both the secreting SAgs and structural component of S. aureus.

The antimicrobial peptides play roles in inhibiting the colonization of $S$. aureus. The colonization of $S$. aureus is inhibited by human $\beta$-defensin-2 (HBD-2) antimicrobial peptide (9). It was reported that the nasal carrier $S$. aureus downregulates the expression of HBD-2 and -3, thereby the carrier strains of $S$. aureus retain an advantage to epithelial colonization and infection (10). It is important to investigate whether $S$. aureus induces the decreased expression of HBD-2, thereby resulting in more susceptible local conditions for colonization of $S$. aureus and persistence of eczema in AD.

Thus, in this study, we tried to isolate and characterize $S$. aureus from an AD patient, with recurrent oozing on the face. We studied the increased expression of inflammatory cytokines, such as IL-1ß, -6, -8, and TNF- $\alpha$ in $S$. aureus treated-HaCaT cells, mediated by secreting SAgs, structural component, or both. In addition, we investigated whether the SAgs from $S$. aureus can down-regulate the expression of HBD-2 in HaCaT cells to make favorable conditions for colonization on the skin.

\section{Materials and methods}

Patient information. The patient, a 17 year-old man, has suffered from AD since childhood. He has dry skin on the trunk with a lichenified patch on the elbow, around the neck, and face. He frequently suffers from recurrent yellowish discharge and oozing of eczematoid on his face. We treated him with saline wet dressing on his face and topical antibiotic creams. This was followed by systemic antibiotics, antihistamines, systemic steroids, and topical steroid creams. 
Isolation and characterization of S. aureus. The bacteria were isolated from eczematous skin lesions of AD by sterile swabs. The bacteria samples were plated into a nonselective blood agar plate and in addition onto the mannitol agar plate containing $7.5 \%$ sodium chloride to improve detection of $S$. aureus. The SAgs toxin genes, such as enterotoxin genes (sea, seb, sec) and exfoliative toxin genes $(e t a, e t b)$ were detected by multiplex PCR as described previously (11). Bacteria supernatants were prepared by growing bacteria in tryptic soy broth (TSB) medium for $16-18 \mathrm{~h}$ at $37^{\circ} \mathrm{C}$ under agitation $(120 \mathrm{rpm})$. Supernatant of $5 \times 10^{8} \mathrm{cfu} / \mathrm{ml}$ at stationary phase was obtained by centrifugation at $960 \mathrm{~g}$ for $10 \mathrm{~min}$ at $4^{\circ} \mathrm{C}$, followed by filtration through a $0.2 \mu \mathrm{m}$ filter. The supernatant corresponding to $S$. aureus soluble SAgs was diluted at $20 \%$ in culture media. TSB medium was used as control at a $20 \%$ dilution in culture media.

Cell culture. Human keratinocyte cell line, HaCaT cells, were maintained at $37^{\circ} \mathrm{C}$ in a humidified atmosphere of $95 \%$ air and $5 \% \mathrm{CO}_{2}$ in Eagle's minimum essential medium supplemented with $10 \%$ heat inactivated fetal bovine serum (FBS), $2 \mathrm{mM}$ glutamine, and $100 \mathrm{U} / \mathrm{ml}$ penicillin and $100 \mu \mathrm{g} / \mathrm{ml}$ streptomycin. For experiments, cells $\left(5 \times 10^{4}\right.$ cells $\left./ \mathrm{ml}\right)$ were seeded in a culture dish and maintained in a tissue culture incubator.

Examination of cell morphology. Cells were fixed with $95 \%$ cold-ethanol for $4 \mathrm{~min}$ and washed twice with PBS. After fixation, cells were stained with staining solution [PI $(1 \mu \mathrm{g} / \mathrm{ml})$, RNase A $(0.1 \mathrm{mg} / \mathrm{ml})]$ for $20 \mathrm{~min}$ at room temperature. Nuclear morphology of PI-stained cells was observed under fluorescence microscope (Olympus Optical, Tokyo, Japan). The cells were also stained with crystal violet solution for $10 \mathrm{~min}$ and washed three times by phosphate buffered saline (PBS) and the morphology of cells was observed under a phase contrast microscope.

Reverse transcription-polymerase chain reaction ( $R T-P C R)$. Total RNA was isolated from cells using the RNAzol ${ }^{\mathrm{TM}} \mathrm{B}$ (Biotecx Laboratories, Houston, TX) according to the manufacturer's instructions and quantitated by spectrophotometer. Total RNA ( $1 \mu \mathrm{g})$ was reverse transcribed using M-MLV reverse transcriptase (Promega Co., Madison, WI). The PCR reaction was carried out following the manufacturer's instructions (Takara Co., Otsu, Japan). The primer sequences and product sizes were as follows: 1) GAPDH (forward, 5'CGT CTT CAC CAC CAT GGA GA-3', reverse, 5'-CGG CCA TCA CGC CAC AGT TT-3'), 300 base pair (bp); 2) IL-13 (forward, 5'- AAA AGC TTG GTG ATG TCT GG-3', reverse, 5'-TTT CAA CAC GCA GGA CAG G-3'), 179 bp; 3) IL-6 (forward, 5'-GTG TGA AAG CAG CAA AGA GGC-3, reverse, 5'-CTG GAG GTA CTC TAG GTA TAC-3'), 159 bp; 4) IL-8 (forward, 5'- ATG ACT TCC AAG CTG GGC CGT G-3', reverse, 5'-TAT GAA TTC TCA GCC CTC TTC AAAA-3'), 301 bp. 5) TNF- $\alpha$ (forward, 5'- CAA AGT AGA CCT GCC CAG AC-3', reverse, 5'-GAC CTC TCT CTA ATC AGC CC-3'), 490 bp. 6) HBD-2 (forward, 5'-ATC TCC TCT TCT CGT TCC TC-3', reverse, 5'-ACC TTC TAG GGC AAA AGA CT-3'), 126 bp.

Western blot analysis. Whole cell extracts were prepared in the lysis buffer [10 mM Tris (pH 7.4), 5 mM EDTA, $130 \mathrm{mM} \mathrm{NaCl}$,

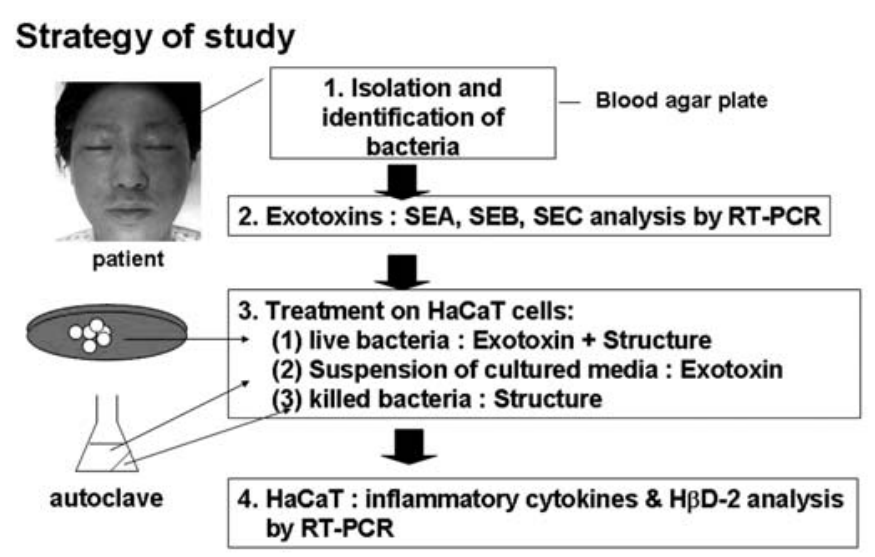

Figure 1. The schematic presentation of this study. The bacteria was isolated from eczematoid skin and characterized by culture media, and the exotoxin genes were specifically amplified by multiplex PCR analysis. The effect of bacteria on expression of inflammatory cytokines and HBD-2 were studied by RT-PCR analysis in live, filtered suspension, or killed bacteria-treated $\mathrm{HaCaT}$ cells.

1\% Triton X-100, phenylmethylsulphonyl fluoride (PMSF), aprotinin, leupeptin (10 $\mathrm{mg} / \mathrm{ml}$ of each), $5 \mathrm{mM}$ phenanthroline and $28 \mathrm{mM}$ benzamidine-HCl]. For phospho-protein detection, cells were washed with ice-cold phosphate-buffered saline containing $1 \mathrm{mM} \mathrm{Na}_{3} \mathrm{VO}_{4}$ and $1 \mathrm{mM} \mathrm{NaF}$, and lysed in a buffer [20 mM Tris- $\mathrm{Cl}$ (pH 8.0), $137 \mathrm{mM} \mathrm{NaCl}, 10 \%$ glycerol, $1 \%$ Triton X-100, $1 \mathrm{mM} \mathrm{Na} \mathrm{VO}_{4}, 1 \mathrm{mM} \mathrm{NaF}, 2 \mathrm{mM}$ EDTA, $200 \mathrm{nM}$ aprotinin, $20 \mu \mathrm{M}$ leupeptin, $50 \mu \mathrm{M}$ phenanthroline, $280 \mu \mathrm{M}$ benzamidine-HCl]. Equal amounts of protein (40 $\mu \mathrm{g} /$ lane) were resolved by $12 \%$ sodium dodecyl sulfatepolyacrylamide gel electrophoresis and transferred onto a nitrocellulose membrane. The membrane was then washed with Tris-buffered saline (10 mM Tris, $150 \mathrm{mM} \mathrm{NaCl})$ containing $0.05 \%$ Tween-20 (TBST) and blocked in TBST containing $5 \%$ non-fat dried milk. The membrane was further incubated with respective specific antibodies such as p-ERK (1:2000), ERK (1:2000), p-JNK (1:1000), JNK (1:2000), p-p38 (1:1000), p38 (1:2000), and $\beta$-tubulin (1:5000). The membrane was continuously incubated with appropriate secondary antibodies coupled with horseradish peroxidase and developed in the ECL Western detection reagents (Amersham Pharmacia Biotech, Piscataway, NJ, USA).

\section{Results}

Isolation of SEA expressing S. aureus. The AD patient clearly showed erythema and eczema with oozing on the face. To isolate and characterize the bacteria on skin lesions, we planned a schematic strategy (Fig. 1) and two swabs were taken according to standard procedures (12). One swab was from an eczematous skin lesion and the other from the nose. The $S$. aureus was isolated from eczematous skin lesion (Fig. 2A) and the isolated S. aureus was of a methicillinsensitive strain (MSSA) (Fig. 2B). The other isolated S. aureus strain from the nose was also MSSA (data not shown). The toxin gene (sea) was detected in isolated $S$. aureus by multiplex PCR but other toxin genes such as seb, sec, and exfoliative toxin genes (eta, etb) were not detected (Fig. 3). To determine the cytotoxic effect of the SEA expressing $S$. 
A

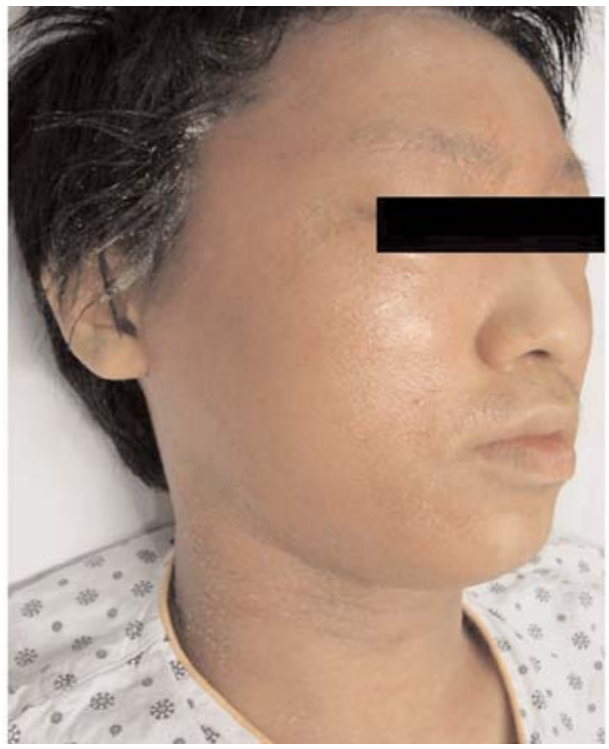

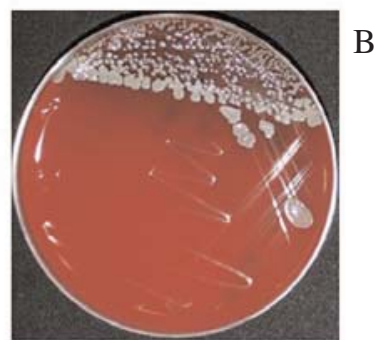

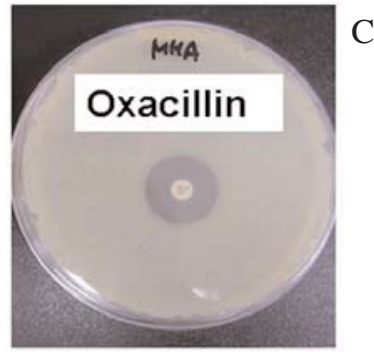

Figure 2. Isolation and culture of S. aureus from an atopic dermatitis patient. (A) Eczematous erythema and yellowish crusts with oozing on the face observed in atopic dermatitis patient. (B) The bacterial colonies were cultured on a blood agar plate and (C) bacteria showed susceptibility to oxacillin.

(A)

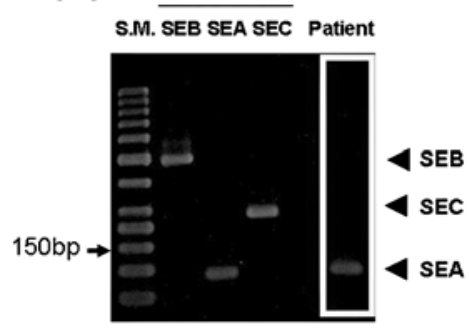

(B)

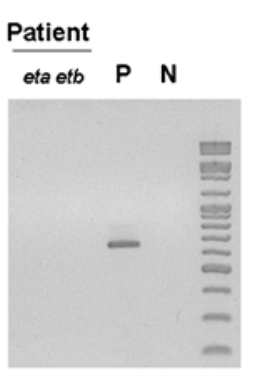

Figure 3. Detection of superantigens and exfoliative toxin genes in S. aureus. (A) The SEA gene, but not SEB and SEC, was clearly amplified by multiplex PCR and (B) both exfoliative toxin genes (ETA and ETB) were not detected by PCR analysis. P, positive control; N, negative control.

aureus, we cocultured HaCaT cells with $S$. aureus. The morphology of HaCaT cells shrank slightly, similar to cytotoxic damaged cells and the fragmented nucleus was observed under a fluorescence microscope (Fig. 4).

Up-regulation of inflammatory cytokines by S. aureus-treated HaCaT cells. To analyze the effect of the structural component of $S$. aureus and SAgs on expression of inflammatory cytokines, we treated live $S$. aureus itself, filtered $(0.02 \mu \mathrm{m}$ acrodisc $)$ supernatant, or killed-S. aureus by autoclave.

First, HaCaT cells were exposed to $S$. aureus and harvested $24 \mathrm{~h}$ after treatment for RT-PCR analysis. As shown in Fig. 5, IL-1ß, $-6,-8$, and TNF- $\alpha$ expressions were dramatically increased by $S$. aureus treatment. To clarify the roles of the
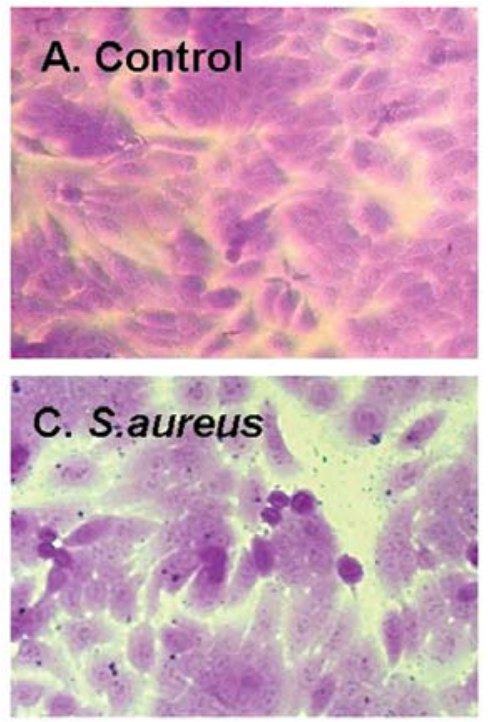
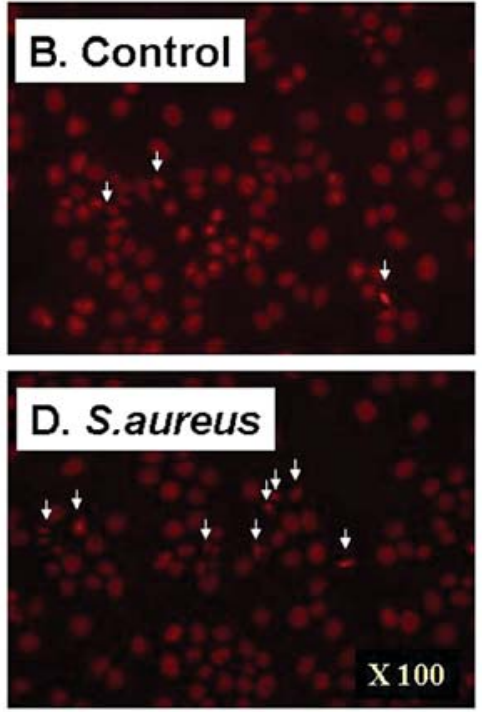

Figure 4. Apoptotic cellular and nuclear morphologies of HaCaT cells. HaCaT cells were cocultured with $S$. aureus in DMEM for 24 h. The morphologies of HaCaT cells cocultured with S. aureus were shrunken and changed to a round shape (arrow), as compared to control (C). The nucleus was markedly fragmented in $\mathrm{HaCaT}$ cells cocultured with $S$. aureus (arrow), as compared to control (D). 


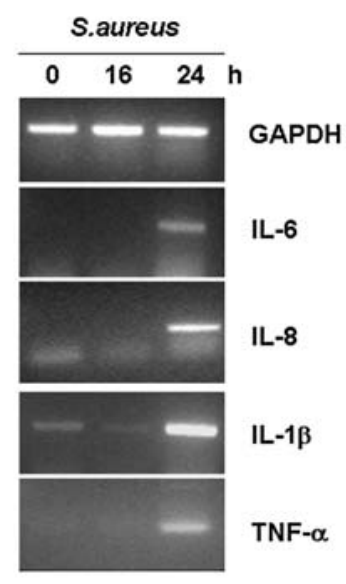

Figure 5. Increased expressions of inflammatory cytokines in $\mathrm{HaCaT}$ cells cocultured with $S$. aureus. The IL-1ß, $-6,-8$, and TNF- $\alpha$ mRNA were specifically amplified by PCR primers.
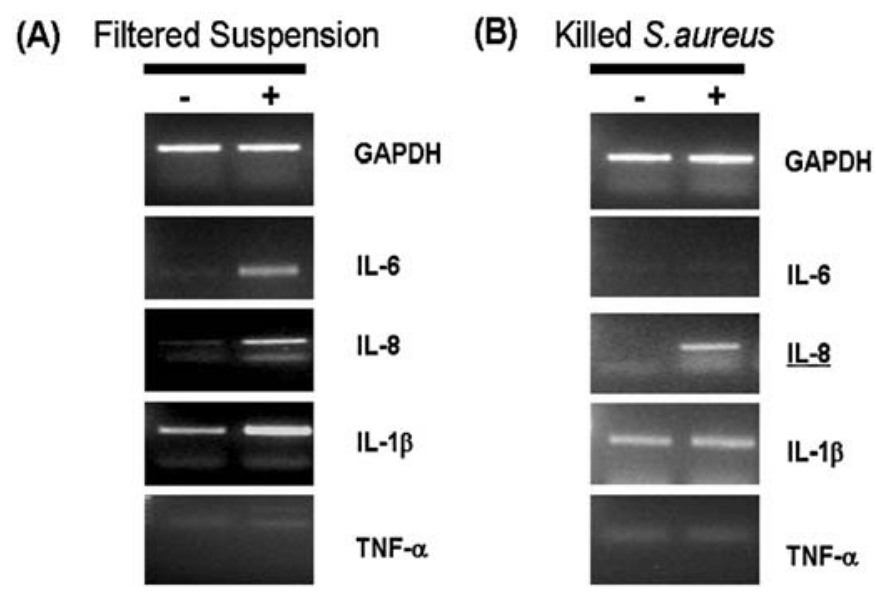

Figure 6. Comparison of expression of inflammatory cytokines between in suspension-treated and autoclaved S. aureus-treated HaCaT cells. (A) $\mathrm{HaCaT}$ cells were treated with culture media of $S$. aureus or (B) autoclaved-S. aureus, and then the IL-1B, $-6,-8$, and TNF- $\alpha$ mRNA were specifically amplified by PCR primers.

secreting SAgs or the structural components of S. aureus on the expression of pro-inflammatory cytokines, $\mathrm{HaCaT}$ cells were treated by suspension of bacterial cultures, filtered by $0.02 \mu \mathrm{m}$ acrodisc, or killed bacteria by autoclave. The expression of IL- $1 \beta,-6$, and -8 , but not TNF- $\alpha$, were increased by the treatment of filtered suspension of bacterial cultures, implying the SAgs induce the expression of IL-1B, -6 , and -8 in HaCaT cells (Fig. 6A). Interestingly, the killed bacteria did not induce the expression of pro-inflammatory cytokines except for IL-8 (Fig. 6B). These results indicate that the increased expression of IL-1ß, -6, -8, and TNF- $\alpha$ in $S$. aureus-exposed HaCaT cells are mainly regulated by the secretion products of $S$. aureus, which may be SAgs (SEA). Further studies are required to elucidate the component of suspension of bacteria, inducing inflammatory cytokine expression.

Up-regulation of HBD-2 by S. aureus-treated HaCaT cells. It is unknown whether the $S$. aureus down-regulates the expression of HBD-2 in keratinocytes to facilitate the skin infection,
(A)

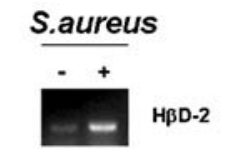

Filtered suspension

killed S.aureus

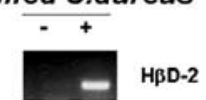

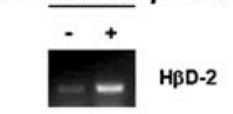

(B)

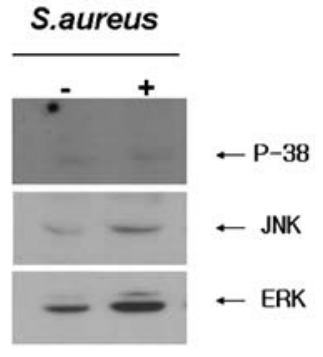

Figure 7. (A) Increased expressions of HBD-2 and (B) activation of JNK and ERK in HaCaT cells by $S$. aureus.

especially in $\mathrm{AD}$ patients showing recurrent erythema and oozing on the face. As shown in Fig. 7A, S. aureus did not down-regulate the expression of HBD-2 in HaCaT cells. We clearly observed that expression of HBD-2 was markedly upregulated in $\mathrm{HaCaT}$ cells by $S$. aureus, implying downregulation of $\mathrm{HBD}-2$ in $\mathrm{AD}$ is regulated by other key factors, but not by $S$. aureus itself.

The expression of HBD-2 is regulated by p38 MAPK or JNK in cells. As shown in Fig. 7B, the activation of JNK and ERK were observed in the $S$. aureus treated HaCaT cells, and stripping and reprobing the same membrane with antibodies against JNK and ERK revealed no change in total protein levels of each kinase (data not shown), indicating that the expression of HBD-2 is regulated partly by the $S$. aureus-induced activation of pre-existing JNK and ERK.

\section{Discussion}

The increased colonization of $S$. aureus in the lesional skin in AD patients contributes to the severity of eczema $(4,5)$. Leung et al reported that $S$. aureus isolated from lesional skin of more than half of their patients with $\mathrm{AD}$ could secrete toxins with the characteristics of SAgs (13). The SAgs of S. aureus are composed of enterotoxins (SEA, SEB, SEC), exofoliative toxins (ETA, ETB), and TSST-1 (14). Furthermore, these SAgs trigger the immunologic responses mediated by cytokines, resulting in inflammation of tissues $(6-8,15)$. Thus, it is accepted that several SAgs are associated with more extensive lesions and the presence of eczema in AD.

In this study, we reported the isolation and characterization of $S$. aureus, which is colonized in the $\mathrm{AD}$ patient showing recurrent eczema on the face for 3 years. The isolated $S$. aureus has the toxin gene, sea, but not seb, sec, eta, and etb. Our data clearly show that the expression of inflammatory cytokines including IL- $1 \beta,-6,-8$, and TNF- $\alpha$, were increased by $S$. aureus. In addition, it was reported that in organ-cultured human skin, the SAgs could induce cytokines (TNF- $\alpha$, IL- $1 \alpha$, and $-1 \beta$ ) and among these cytokines, TNF- $\alpha$ was the most abundantly expressed in the keratinocytes at both the protein and mRNA levels (16). Both IL-1ß and TNF- $\alpha$ play roles as potent primary stimuli for IL- 6 and -8 production (17). The local cytokine levels of TNF- $\alpha$, IL-1ß, -6 and -8 were markedly high in severe exanthematous diseases in newborn infants caused by exotoxins producing methicillin-resistant $S$. aureus 
(18). Interestingly, in this study, our data show that the expression of IL-8, but not IL-1ß, TNF- $\alpha$, and IL-6, was upregulated by the treatment of autoclaved-S. aureus. These data indicate that the expression of IL- 8 is regulated by the structure of $S$. aureus as well as secretion products such as exotoxins. It was reported that lipoteichoic acid from $S$. aureus induced a strong release of the chemoattractants, LTB4, IL-8, C5a, MCP-1, and the colony-stimulating factor G-CSF in whole blood, playing roles in stimulus for neutrophil recruitment (19). Taken together, the SAgs and structure of $S$. aureus such as lipoteichoic acid have been shown to exert direct pro-inflammatory effects on keratinocytes via TNF- $\alpha$ or IL-1ß secretion, resulting in induction and persistent eczematous skin lesions in AD.

The AD patients are highly susceptible to cutaneous bacterial, fungal, and viral infections $(1,2)$. The decreased expression of antimicrobial peptides, such as HBD-2 and -3, partly play roles in the underlying molecular mechanisms of the increased susceptibility of bacterial cutaneous infections in AD (9). However, the precise molecular mechanisms of the decreased expression of HBD-2 in AD are still unknown. Recently, it was reported that the nasal carrier $S$. aureus delayed the expression of the pattern recognition receptor, Toll-like receptor 2 (TLR2) and furthermore down-regulates the expression of HBD-2 and -3, thereby the carrier strains of $S$. aureus retain an advantage by delaying the host's innate response to epithelial colonization and infection (10). Our patient also had nasal $S$. aureus, producing the SAgs, and showed recurrent eczematous skin lesions on the face. Thus, we hypothesized that the colonization of the SAgproducing $S$. aureus down-regulates the expression of HBD-2 in AD for facilitating the cutaneous skin. In this study, our data clearly show that the expression of HBD-2 was markedly increased by the live and cultured media of $S$. aureus, or killed S. aureus-treated HaCaT cells. Thus, SAgproducing $S$. aureus did not down-regulate the expression of HBD-2 in HaCaT cells. Recently, it was suggested that the increased expression of IL-4, -13, and -10 in AD lesions may contribute to the decreased expression of HBD-2 by downregulation of cytokine expression, which has the capability to induce the HBD-2 expression. To clarify the roles of SAgproducing $S$. aureus in eczema, further studies should be performed among the expression levels of pro-inflammatory cytokines, HBD-2, and colonization density of SAgproducing $S$. aureus in eczematoid skin in AD.

In conclusion, we demonstrated the SAg-producing $S$. aureus from an $\mathrm{AD}$ patient with recurrent oozing on the face. The same strain was also colonized in the nostril of the AD patient. SAg-producing $S$. aureus induce pro-inflammatory cytokines, such as IL-1ß, -6 , and -8 as well as HBD-2 in HaCaT keratinocytes through secretion of SEA, thereby resulting in induction and persistent eczematous skin lesion in AD. Furthermore, the expression of IL- 8 is also induced by the structure component of $S$. aureus. Thus, our data may give insight into understanding the pathogenesis by which $S$. aureus induces and aggravates eczematous skin lesions in AD.

\section{References}

1. Bellanti JA and Settipane RA: Atopic Dermatitis, Genetics, Immunology, and T regulatory cells. Allergy Asthma Proc 28: 507-509, 2007.

2. Giannetti A and Girolomoni G: Skin diseases with high public health impact. Atopic dermatitis. Eur J Dermatol 17: 566, 2007.

3. Levy ML: Atopic dermatitis: understanding the disease and its management. Curr Med Res Opin 23: 3091-3103, 2007.

4. Gong JQ, Lin L, Lin T, Hao F, Zeng FQ, Bi ZG, Yi D and Zhao B: Skin colonization by Staphylococcus aureus in patients with eczema and atopic dermatitis and relevant combined topical therapy: a double-blind multicentre randomized controlled trial. Br J Dermatol 155: 680-687, 2006.

5. Baker BS: The role of microorganisms in atopic dermatitis. Clin Exp Immunol 144: 1-9, 2006.

6. Davison S, Allen M, Vaughan R and Barker J: Staphylococcal toxin-induced $\mathrm{T}$ cell proliferation in atopic eczema correlates with increased use of superantigen-reactive Vbeta-chains in cutaneous lymphocyte-associated antigen (CLA)-positive lymphocytes. Clin Exp Immunol 121: 181-186, 2000.

7. Homey B, Meller S, Savinko T, Alenius H and Lauerma A: Modulation of chemokines by staphylococcal superantigen in atopic dermatitis. Chem Immunol Allergy 93: 181-194, 2007.

8. Kim KH, Han JH, Chung JH, Cho KH and Eun HC: Role of staphylococcal superantigen in atopic dermatitis: influence on keratinocytes. J Korean Med Sci 21: 315-323, 2006.

9. Ong PY, Ohtake T, Brandt C, Strickland I, Boguniewicz M, Ganz T, Gallo RL and Leung DY: Endogenous antimicrobial peptides and skin infections in atopic dermatitis. N Engl J Med 347: 1151-1160, 2002.

10. Quinn GA and Cole AM: Suppression of innate immunity by a nasal carriage strain of Staphylococcus aureus increases its colonization on nasal epithelium. Immunology 122: 80-89, 2007.

11. Becker K, Roth R and Peters G: Rapid and specific detection of toxigenic Staphylococcus aureus: use of two multiplex PCR enzyme immunoassays for amplification and hybridization of staphylococcal enterotoxin genes, exfoliative toxin genes, and toxic shock syndrome toxin 1 gene. J Clin Microbiol 36: 2548-2553, 1998.

12. Murray PR, Baron EJ, Jorgensen JH, Landry ML, Pfaller MA, and Stratton C: Manual of clinical microbiology, 9th edition: manual of clinical microbiology, 9th edition. Clin Infect Dis 46: 153-154, 2008

13. Leung DY, Harbeck R, Bina P, Reiser RF, Yang E, Norris DA, Hanifin JM and Sampson HA: Presence of IgE antibodies to staphylococcal exotoxins on the skin of patients with atopic dermatitis. Evidence for a new group of allergens. J Clin Invest 92: 1374-1380, 1993

14. Lin YT, Wang CT and Chiang BL: Role of bacterial pathogens in atopic dermatitis. Clin Rev Allergy Immunol 33: 167-177, 2007.

15. Herz U, Bunikowski R and Renz H: Role of T cells in atopic dermatitis. New aspects on the dynamics of cytokine production and the contribution of bacterial superantigens. Int Arch Allergy Immunol 115: 179-190, 1998.

16. Matsunaga T, Katayama I, Yokozeki H and Nishioka K: Superantigen-induced cytokine expression in organ-cultured human skin. J Dermatol Sci 11: 104-110, 1996.

17. Soderquist B, Kallman J, Holmberg H, Vikerfors T and Kihlstrom E: Secretion of IL-6, IL-8 and G-CSF by human endothelial cells in vitro in response to Staphylococcus aureus and staphylococcal exotoxins. APMIS 106: 1157-1164, 1998

18. Okada T, Makimoto A, Kitamura A, Furukawa S, Miwa K and Sakai R: [A new exanthematous disease in newborn infants caused by exotoxins producing methicillin-resistant Staphylococcus aureus; pathology from viewpoints of local and systemic levels of exotoxin and cytokine]. Kansenshogaku Zasshi 74: 573-579, 2000.

19. Von Aulock S, Morath S, Hareng L, Knapp S, van Kessel KP, van Strijp JA and Hartung T: Lipoteichoic acid from Staphylococcus aureus is a potent stimulus for neutrophil recruitment. Immunobiology 208: 413-422, 2003. 